\title{
Constrained sliding mode control of nonlinear fractional order input affine systems
}

\author{
TAHMINEH Vedadi Moghaddam, SEYYED KAMALEDDIN Yadavar Nikravesh, and \\ MOHAMMAD Azam Khosravi*
}

Electrical Engineering Department, Amirkabir University of Technology, Tehran 158754413, Iran

\begin{abstract}
Asymptotic stability of nonlinear fractional order affine systems with bounded inputs is dealt. The main contribution is to design a new bounded fractional order chattering free sliding mode controller in which the system states converge to the sliding surface at a determined finite time. To eliminate the chattering in the sliding mode and make the input controller bounded, hyperbolic tangent is used for designing the proposed fractional order sliding surface. Finally, the stability of the closed loop system using this bounded sliding mode controller is guaranteed by Lyapunov theory. A comparison with the integer order case is then presented and fractional order nonlinear polynomial systems are also studied as the special case. Finally, simulation results are provided to show the effectiveness of the designed controller.
\end{abstract}

Keywords: constrained sliding mode control, nonlinear fractional order systems, input affine systems.

DOI: $10.21629 / J S E E .2019 .05 .16$

\section{Introduction}

In spite of great efforts that have been done in control of fractional order systems, there are still insignificant researches on how to apply these controllers for fractional order systems with non-smooth non-linearity or in the presence of constraint in their inputs. As it is obvious, input constraints such as input saturations are common in industrial applications [1]. In practical applications, system performance becomes difficult due to the input limitations. In the integer order of such systems, some classical controllers such as back-stepping have been employed [2-9]. In [10], discrete-time low-gain adaptive and non-adaptive control strategies were presented for tracking constant reference signals for infinite dimensional linear systems subject to input nonlinearities. An input to the state stability method for hybrid systems with the input constraint was presented in [11].

For fractional order systems subject to the input con-

Manuscript received July 18, 2018.

*Corresponding author. straint, the change in system order eliminates the effectiveness of proposed control methods. Nevertheless, several control methods have been provided for these systems in recent years but each of them has its own disadvantages and limitations. For instances, in [12], Lim et al. introduced a method using sector bounded condition and Gronwall-Bellman lemma that asymptotically will stabilize linear fractional order systems subject to input saturation. In [13,14], Shahri et al. presented the stability analysis of the same system with the direct Lyapunov method and also studied the disturbance rejection for these kinds of systems. In [15], Luo considered the nonlinear fractional order system with input saturation but the nonlinear function is limited to piecewise continuous functions that satisfy locally Lipchitz conditions. Separated from all the above mentioned papers, there are still many deficiencies in the previous works. Considering some restrictions on the system input and designing a more effective controller other than classical linear controllers can be an effective progress in this field.

On the other hand, to deal with the uncertainties and external disturbances, robust control methods have attracted widespread attention and been developed in many directions of control science. There are several robust methods in this area including optimal control, H-infinity control, high gain feedback control, variable structure control, etc. One of the new and straight ideas in this field is sliding mode control. Researches in the field of sliding mode control began about 50 years ago and over the last two decades this control method has attracted more attention in the international control community. One of the most fascinating aspects of the sliding mode is the discontinuous nature of control performance, so its initial function switches between two different structures. This nature of sliding mode control is also used to overcome uncertainties and external disturbances in the system. Due to this features, the studies on the siding mode control in the field of industrial applica- 
tions or academic studies have been extensively increased. Nowadays the sliding mode control method is applicable on a wide range of systems such as robotic, electric drives, generators, process control, vehicle control, motion control, fractional-order systems and so on.

Sliding mode controllers are used to improve the efficiency and robustness of closed loop systems [16]. Sliding mode control has effective features such as immutability to uncertainties, simplicity of design, robustness to perturbation and some other properties [17-19]. The specific feature of continuous time sliding mode control is that the sliding mode will happen in a predetermined sliding surface and then the switching control is applied to the system to force the states to remain in the sliding surface [20]. Generally, any linear sliding surface can guarantee the asymptotical stability and getting desired performance in a closed loop system. However, the monotonous switching feedback control gain increases the settling time of the closed loop system which means that the system states cannot converge to the equilibrium point in a finite time. In addition, sliding mode control makes the control input have high frequency chattering which causes undesirable load on control operators.

During the development of fractional order control, many researches have tried to use the fractional order sliding mode control to overcome the above-mentioned problems. In [21], Dadras et al. introduced a new control strategy for a class of uncertain dynamical systems called fractional order terminal sliding mode control. Based on the Lyapunov stability theorem, fractional order switching manifold is provided to assurance of sliding condition. In [22], Majumdar et al. presented a fractional order sliding mode control for controlling a single link flexible manipulator. Also, switching surface is obtained based on fractional order derivatives of related differential equations. The proposed fractional order sliding mode controller makes better performance with fewer fluctuation in the input control and it is robust against external disturbances.

In [23], Zhang et al. provided an optimal fractional order sliding mode control strategy for a class of nonlinear systems in the presence of uncertainty. This proposed control strategy is based on the linear quadratic optimal theory. In particular, a new fractional order sliding surface is provided based on the linear quadratic regulator. The provided controller in this method has integer and fractional order parts. In the proposed controller, fast states convergence is on the integer order part and system robustness against uncertainties is on the fractional order part. In the context of controlling the fractional order systems, sliding mode control was also presented in [24,25] and higher order sliding mode control was also expressed in [26].
To the author's best knowledge, constrained sliding mode control for nonlinear fractional order input affine systems has not been studied yet. Therefore, this paper will propose a constrained chattering free sliding mode control for stabilizing the nonlinear fractional order input affine systems that are subjected to input constraints. Using this sliding mode controller, the system states converge to the sliding surface in a pre-described finite time and the controller will guarantee the asymptotically stability of the closed loop system based on the Lyapunov theory.

The rest of this paper is organized as follows: in Section 2, the problem formulation will be expressed. Then, in Section 3, design of the constrained chattering free sliding mode controller is provided and fractional order nonlinear polynomial systems are also studied as a special case of these systems. In Section 4, the finite time convergence of the sliding surface to zero is determined. A comparison between the effectiveness of the constrained fractional order controller and its integer order counterpart in confronting of chattering is discussed in Section 5. Finally, in Section 6, numerical examples with simulation results will show the validity of the proposed method.

\section{Problem formulation}

A nonlinear fractional order (NFO) input affine system is shown with the following general form:

$$
{ }_{0} D_{t}^{\alpha} \boldsymbol{x}(t)=f(\boldsymbol{x}(t))+g(\boldsymbol{x}(t))(\boldsymbol{u}(t))
$$

where $f(\boldsymbol{x}(t)) \in \mathbf{R}^{n \times 1}$ and $g(\boldsymbol{x}(t)) \in \mathbf{R}^{n \times m}$ are arbitrary nonlinear functions and $f(\boldsymbol{x}(t))$ satisfies the Lipchitz condition, $\boldsymbol{x}(t) \in \mathbf{R}^{n}$ is the pseudo state vector, $\boldsymbol{u}(t) \in \mathbf{R}^{m}$ is the input control signal, $0<\alpha<1$ is the fractional order of system and ${ }_{0} D_{t}^{\alpha}$ denotes the Caputo fractional derivative operator that is defined for function $\boldsymbol{x}(t)[27]$ as bellow:

$$
\begin{gathered}
{ }_{0} D_{t}^{\alpha} \boldsymbol{x}(t)=\frac{1}{\Gamma(\lceil\alpha\rceil-\alpha)} \int_{0}^{t} \frac{\boldsymbol{x}^{(\lceil\alpha\rceil)}(\tau)}{(t-\tau)^{\alpha-\lceil\alpha\rceil+1}} \mathrm{~d} \tau \\
0<\alpha \notin \mathbf{Z}_{+}
\end{gathered}
$$

where $\Gamma(\cdot)$ denotes the Gamma function and $\lceil\alpha\rceil$ maps $\alpha$ to the least integer that is greater than or equal to $\alpha$ [27].

The objective of this paper is to design a new constrained sliding mode controller $\boldsymbol{u}(t) \in \mathbf{R}^{m}$ for the NFO input affine system in (1) in such a way that asymptotic stability is guaranteed with specific finite time convergence to the switching manifold and without any chattering. First, the asymptotic stability of an NFO system is defined as follows.

Definition 1 (Stability of NFO systems) [27] The zero solution of ${ }_{0} D_{t}^{\alpha} \boldsymbol{x}(t)=f(\boldsymbol{x}(t))$ is said to be stable if, for any initial conditions $\boldsymbol{x}(0) \in \mathbf{R}^{n}$, there exists $\delta>0$ such that any solution $\boldsymbol{x}(t)$ of ${ }_{0} D_{t}^{\alpha} \boldsymbol{x}(t)=f(\boldsymbol{x}(t))$ satisfies 
$\|\boldsymbol{x}(t)\|<\delta$ for all $t>t_{0}$. Further, the zero solution of the fractional system is said to be asymptotically stable if the system is stable and $\|\boldsymbol{x}(t)\| \rightarrow 0$ as $t \rightarrow \infty$.

Using the above mentioned stability definition, a nonlinear stable function is also defined as follows.

Definition 2 (Stability of nonlinear functions) A nonlinear vector function $L(\boldsymbol{x}) \in \mathbf{R}^{n \times 1}$ is said to be asymptotically stable if and only if the NFO system ${ }_{0} D_{t}^{\alpha} \boldsymbol{x}(t)=$ $L(\boldsymbol{x}(t))$ in (1) is asymptotically stable.

\section{Constrained sliding mode controller design for NFO input affine system}

In this section the design procedure of the constrained sliding mode controller for the NFO input affine system in (1) is provided. To this end, at first using the tangent hyperbolic function which is a continuous and bounded function, a new fractional-integral sliding surface is designed and after that the main controller that is a constrained sliding mode controller is constructed.

\subsection{Design of the sliding manifold}

One of the main purposes in this paper is to design a controller that is bounded. Therefore, the controller must be designed in such a way that the system input remains limited. For this aim, a new sliding surface is designed as follows:

$$
\begin{gathered}
\boldsymbol{S}(t)=\tanh \left(\int_{0}^{t} h(\boldsymbol{x}(\tau))_{0} D_{t}^{\alpha} \boldsymbol{x}(\tau) \mathrm{d} \tau-\right. \\
\left.\int_{0}^{t} h(\boldsymbol{x}(\tau))(f(\boldsymbol{x}(\tau))+L(\boldsymbol{x}(\tau))) \mathrm{d} \tau\right)
\end{gathered}
$$

where $\boldsymbol{S}(t)=\left[s_{1}, s_{2}, \ldots, s_{m}\right] \in \mathbf{R}^{m}$ is the sliding manifold vector, $\tanh (\cdot)$ is applied to all the elements of $\boldsymbol{S}(t)$, $h(\boldsymbol{x}) \in \mathbf{R}^{m \times n}$ and $L(\boldsymbol{x}) \in \mathbf{R}^{n \times 1}$ are arbitrary nonlinear functions that are considered as control parameters and should satisfy the following assumption.

Assumption 1 Suppose the nonlinear vector functions $h(\boldsymbol{x}) \in \mathbf{R}^{m \times n}$ and $L(\boldsymbol{x}) \in \mathbf{R}^{n \times 1}$ satisfy the following conditions:

(i) $\forall \boldsymbol{x} \in \mathbf{R}^{n \times 1} h(\boldsymbol{x}) \neq 0$.

(ii) $h(\boldsymbol{x}) g(\boldsymbol{x}) \in \mathbf{R}^{m \times m}$ is nonsingular.

(iii) $(h(\boldsymbol{x}(t)) g(\boldsymbol{x}(t)))^{-1} \in \mathbf{R}^{m \times m}$ and $h(\boldsymbol{x}(t)) L(\boldsymbol{x}(t)) \in$ $\mathbf{R}^{m \times 1}$ are bounded, i.e., $\forall \boldsymbol{x} \in \mathbf{R}^{n \times 1}\left\|(h(\boldsymbol{x}(t)) g(\boldsymbol{x}(t)))^{-1}\right\|$ $\leqslant M$ and $\|h(\boldsymbol{x}(t)) L(\boldsymbol{x}(t))\| \leqslant N$.

(iv) $f(x(\boldsymbol{t}))+L(\boldsymbol{x}(t))$ is asymptotically stable.

Remark 1 As it is clear, the defined sliding manifold in (3) consists of a tangent hyperbolic function. Therefore, all the elements of this sliding surface are bounded between -1 and 1 . In addition, despite of the saturation or the sign function, this function is a continuous function.

According to the sliding mode approach, the switching manifold and its derivative should be equal to zero. Taking the derivative of ( 3 ) the following equation is obtained:

$$
\begin{gathered}
\dot{\boldsymbol{S}}(t)=\left(h(\boldsymbol{x}(t))_{0} D_{t}^{\alpha} \boldsymbol{x}(t)-h(\boldsymbol{x}(t))(f(\boldsymbol{x}(t))+L(\boldsymbol{x}(t))) \times\right. \\
\operatorname{sech}^{2}\left(\int_{0}^{t} h(\boldsymbol{x}(\tau))_{0} D_{t}^{\alpha} \boldsymbol{x}(\tau) \mathrm{d} \tau-\right. \\
\left.\int_{0}^{t} h(\boldsymbol{x}(\tau))(f(\boldsymbol{x}(\tau))+L(\boldsymbol{x}(\tau))) \mathrm{d} \tau\right) .
\end{gathered}
$$

Now by defining

$$
\begin{gathered}
\boldsymbol{P}(t)=\left(\int_{0}^{t} h(\boldsymbol{x}(\tau))_{0} D_{t}^{\alpha} \boldsymbol{x}(\tau) \mathrm{d} \tau-\right. \\
\left.\int_{0}^{t} h(\boldsymbol{x}(\tau))(f(\boldsymbol{x}(\tau))+L(\boldsymbol{x}(\tau))) \mathrm{d} \tau\right)
\end{gathered}
$$

where $\boldsymbol{P}(t)=\left[p_{1}, p_{2}, \ldots, p_{m}\right] \in \mathbf{R}^{m}$, the sliding manifold in (3) can be rewritten as $\boldsymbol{S}(t)=\tanh (\boldsymbol{P}(t))$ and consequently

$$
\boldsymbol{P}(t)=\tanh ^{-1}(\boldsymbol{S}(t))
$$

which means that $p_{i}=\tanh ^{-1}\left(s_{i}\right)$ for $i=1,2, \ldots, m$.

Now, using (5) and (6), (4) can be rewritten as

$$
\begin{gathered}
\dot{\boldsymbol{S}}(t)=\left(h(\boldsymbol{x}(t))_{0} D_{t}^{\alpha} \boldsymbol{x}(t)-h(\boldsymbol{x}(t))(f(\boldsymbol{x}(t))+\right. \\
L(\boldsymbol{x}(t))) \times \operatorname{sech}^{2}\left(\tanh ^{-1}(\boldsymbol{S}(t))\right) .
\end{gathered}
$$

In the sliding mode situation, the switching manifold and its derivative are equal to zero $(\boldsymbol{S}(t)=0, \dot{\boldsymbol{S}}(t)=0)$ and from (7) we have

$$
\begin{gathered}
\left(h(\boldsymbol{x}(t))_{0} D_{t}^{\alpha} \boldsymbol{x}(t)-h(\boldsymbol{x}(t))(f(\boldsymbol{x}(t))+L(\boldsymbol{x}(t))) \times\right. \\
\operatorname{sech}^{2}\left(\tanh ^{-1}(\boldsymbol{S}(t))\right)=0 \\
\Rightarrow h(\boldsymbol{x}(t))\left({ }_{0} D_{t}^{\alpha} \boldsymbol{x}(t)-f(\boldsymbol{x}(t))-L(\boldsymbol{x}(t))\right) \times \\
\operatorname{sech}^{2}\left(\tanh ^{-1}(\boldsymbol{S}(t))\right)=0 .
\end{gathered}
$$

Now, given that $h(\boldsymbol{x}(t)) \neq 0$ (item (i) of Assumption 1) and considering the equation $\operatorname{sech}^{2}\left(\tanh ^{-1}(\boldsymbol{S}(t))\right)=1$ for $\boldsymbol{S}(t)=0$, (8) can be written as below:

$$
{ }_{0} D_{t}^{\alpha} \boldsymbol{x}(t)=f(\boldsymbol{x}(t))+L(\boldsymbol{x}(t)) .
$$

Finally, given that $f(\boldsymbol{x}(t))+L(\boldsymbol{x}(t))$ is asymptotically stable (item (iv) of Assumption 1), $\boldsymbol{x}(t)$ converges to zero where $t \rightarrow \infty$.

Using the sliding manifold in (3), a bounded sliding mode controller is suggested in the following subsection.

\subsection{Constrained sliding mode control}

In this subsection, the constrained sliding mode control law is suggested as follows:

$$
\begin{gathered}
\boldsymbol{u}(t)=(h(\boldsymbol{x}(t)) g(\boldsymbol{x}(t)))^{-1}(h(\boldsymbol{x}(t)) L(\boldsymbol{x}(t))- \\
\mu \boldsymbol{S}(t)-\rho \operatorname{sgn}(\boldsymbol{S}(t)))
\end{gathered}
$$


where $\boldsymbol{u}(t)=\left[u_{1}, \ldots, u_{m}\right]^{\mathrm{T}} \in \mathbf{R}^{m}$ and $\mu, \rho \in \mathbf{R}^{+}$are positive real constants.

Before expressing the stability analysis of the sliding mode controller in (10), we want to show that this suggested input control is bounded.

At first, it is worth mentioning that according to the definition of $\boldsymbol{S}(t)$ in (3) and $\boldsymbol{P}(t)$ in (5) we have

$$
\begin{gathered}
\|\boldsymbol{S}(t)\|=\sqrt{\left\|s_{1}\right\|^{2}+\left\|s_{2}\right\|^{2}+\cdots+\left\|s_{m}\right\|^{2}}= \\
\sqrt{\left\|\tanh \left(p_{1}\right)\right\|^{2}+\left\|\tanh \left(p_{2}\right)\right\|^{2}+\cdots+\left\|\tanh \left(p_{m}\right)\right\|^{2}} .
\end{gathered}
$$

Now, given that $\left|\tanh \left(p_{i}\right)\right| \leqslant 1, i=1,2, \ldots, m$, the upper bound of sliding manifold $\boldsymbol{S}(t)$ in (11) is obtained as follows:

$$
\|\boldsymbol{S}(t)\| \leqslant \sqrt{\underbrace{1+1+\cdots+1}_{m \text { time }}}=\sqrt{m} .
$$

Then, due to the definition of the sign function, the similar calculations will also result in

$$
\|\operatorname{sgn}(\boldsymbol{S}(t))\| \leqslant \sqrt{m} .
$$

Therefore, the upper bound of the input control law in (10) can be obtained as below:

$$
\begin{gathered}
\|\boldsymbol{u}(t)\| \leqslant\left\|(h(\boldsymbol{x}(t)) g(\boldsymbol{x}(t)))^{-1}\right\| \|(h(\boldsymbol{x}(t)) L(\boldsymbol{x}(t))- \\
\mu \boldsymbol{S}(t)-\rho \operatorname{sgn}(\boldsymbol{S}(t))) \| .
\end{gathered}
$$

Therefore, due to the positivity of $\mu$ and $\rho$ and considering (12) and (13), the upper bound of input control will be achieved as follows:

$$
\begin{gathered}
\|\boldsymbol{u}(t)\| \leqslant\left\|(h(\boldsymbol{x}(t)) g(\boldsymbol{x}(t)))^{-1}\right\|\{\|h(\boldsymbol{x}(t)) L(\boldsymbol{x}(t))\|+ \\
\mu \sqrt{m}+\rho \sqrt{m}\} .
\end{gathered}
$$

Finally, considering the inequalities mentioned in item (iii) of Assumption 1, the upper bound of the input control is obtained as below:

$$
\|\boldsymbol{u}(t)\| \leqslant M\{N+\mu \sqrt{m}+\rho \sqrt{m}\} .
$$

Now, we are ready to show the stabilizing property of the constraint input controller in (10) that is done in the next subsection.

\subsection{Stability analysis}

The main result of the paper is expressed in this subsection. A complete description of the proposed sliding mode controller for asymptotically stabilizing of the NFO input affine system in (1) is presented in the following theorem.
Theorem 1 Consider the NFO input affine in (3). Let $\boldsymbol{u}(t)=\left[u_{1}, \ldots, u_{m}\right]^{\mathrm{T}} \in \mathbf{R}^{m}$ is given by (10) where $h(\boldsymbol{x}) \in \mathbf{R}^{m \times n}$ and $L(\boldsymbol{x}) \in \mathbf{R}^{n \times 1}$ are arbitrary nonlinear functions that satisfy Assumption $1, \boldsymbol{S}(t)$ is defined by (3) and $\mu, \rho>0$. Then,

(i) the closed loop system is asymptotically stable,

(ii) input control in (10) is bounded and its upper bound is shown in (16).

Proof Consider the following Lyapunov function candidate:

$$
V(t)=\frac{1}{2} \boldsymbol{S}^{\mathrm{T}}(t) \boldsymbol{S}(t) .
$$

Taking derivative of (17) along the trajectories of system in (1) results in

$$
\begin{gathered}
\dot{V}(t)=\boldsymbol{S}^{\mathrm{T}}(t) \dot{\boldsymbol{S}}(t)= \\
\boldsymbol{S}^{\mathrm{T}}(t)\left(h(\boldsymbol{x}(t)) D^{\alpha} \boldsymbol{x}(t)-h(\boldsymbol{x}(t)) f(\boldsymbol{x}(t))-\right. \\
h(\boldsymbol{x}(t)) L(\boldsymbol{x}(t))) \times\left(\operatorname{sech}^{2}\left(\tanh ^{-1}(\boldsymbol{S}(t))\right)\right)= \\
\boldsymbol{S}^{\mathrm{T}}(t)(h(\boldsymbol{x}(t)) g(\boldsymbol{x}(t)) \boldsymbol{u}(t)-h(\boldsymbol{x}(t)) L(\boldsymbol{x}(t))) \times \\
\left(\operatorname{sech}^{2}\left(\tanh ^{-1}(\boldsymbol{S}(t))\right)\right) .
\end{gathered}
$$

Substituting the input controller from (10) into (18) results in

$$
\begin{aligned}
\dot{V}(t)= & \boldsymbol{S}^{\mathrm{T}}(t)(-\mu \boldsymbol{S}(t)-\rho \operatorname{sgn}(\boldsymbol{S}(t))) . \\
& \left(\operatorname{sech}^{2}\left(\tanh ^{-1}(\boldsymbol{S}(t))\right)\right) .
\end{aligned}
$$

That can be written as

$$
\dot{V}(t)=-\mu\|\boldsymbol{S}(t)\|^{2}-\rho\|\boldsymbol{S}(t)\|\left(\operatorname{sech}^{2}\left(\tanh ^{-1}(\boldsymbol{S}(t))\right)\right) \text {. }
$$

Therefore, due to the positivity of $\mu$ and $\rho$, the derivative of the Lyapunov function in (20) is negative definite and thus $\boldsymbol{S}(t)$ converges to zero where $t \rightarrow \infty$. Therefore, according to (9), the system in (1) with the control input in (10) is asymptotically stable.

\subsection{Special case}

Suppose that system functions $f(x)$ and $g(x)$ in fractional order system in (1) are in scalar polynomial forms as follows:

$$
\left\{\begin{array}{l}
f(x(t))=a_{n} x^{n}+a_{n-1} x^{n-1}+\cdots+a_{1} x+a_{0} \\
g(x(t))=b_{m} x^{m}+b_{m-1} x^{m-1}+\cdots+b_{1} x+b_{0}
\end{array}\right.
$$

where $a_{i}>0(i=1, \ldots, n)$ and $b_{j}>0(j=1, \ldots, m)$ are constant coefficients.

It is obvious that $f(x)$ has $n$ roots which can be displayed with $\lambda_{i}(i=1, \ldots, n)$. It is known that a stable root in the fractional order system is in the region $\left|\arg \left(\lambda_{i}\right)\right|>\alpha \frac{\pi}{2}$, where $\alpha$ is the fractional order. Now suppose that $f(x)$ has $p$ stable roots and $n-p$ unstable roots. 
Then, by separating the stable and unstable parts, $f(x)$ can be rewritten as follows:

$$
\begin{gathered}
f(x(t))=\underbrace{\left(x-\lambda_{1}\right)\left(x-\lambda_{2}\right) \cdots\left(x-\lambda_{p}\right)}_{p \text { stable roots }} . \\
\underbrace{\left(x-\lambda_{p+1}\right)\left(x-\lambda_{p+2}\right) \cdots\left(x-\lambda_{n}\right)}_{(n-p) \text { unstable roots }} .
\end{gathered}
$$

According to item (iv) of Assumption 1, $f(x)+L(x)$ is asymptotically stable therefore $L(x)$ could be designed as follows:

$$
f(x(t))+L(x(t))=\left(x-\lambda_{1}\right)\left(x-\lambda_{2}\right) \cdots\left(x-\lambda_{p}\right) .
$$

Therefore,

$L(x(t))=\left(x-\lambda_{1}\right) \cdots\left(x-\lambda_{p}\right)\left(1-\left(x-\lambda_{p+1}\right) \cdots\left(x-\lambda_{n}\right)\right)$.

$$
\begin{gathered}
L(x(t))=\left(x^{p}-\left(\sum_{i=1}^{p} \lambda_{i}\right) x^{p-1}+\left(\sum_{\substack{i, j=1 \\
i \neq j}}^{p} \lambda_{i} \lambda_{j}\right) x^{p-2}-\left(\sum_{\substack{i, j, k=1 \\
i \neq j \neq k}}^{p} \lambda_{i} \lambda_{j} \lambda_{k}\right) x^{p-3}+\cdots+(-1)^{p} \lambda_{1} \lambda_{2} \cdots \lambda_{p}\right) \times \\
\left(1-\left(x^{n-p}-\left(\sum_{i=1}^{n-p} \lambda_{i}\right) x^{n-p-1}+\left(\sum_{\substack{i, j=1 \\
i \neq j}}^{n-p} \lambda_{i} \lambda_{j}\right) x^{n-p-2}-\left(\sum_{\substack{i, j, k=1 \\
i \neq j \neq k}}^{n-p} \lambda_{i} \lambda_{j} \lambda_{k}\right) x^{n-p-3}+\cdots+(-1)^{n-p} \lambda_{p+1} \lambda_{p+2} \cdots \lambda_{n}\right)\right),
\end{gathered}
$$

which can be simplified as follows:

$$
\begin{gathered}
L(x(t))=-\left(x^{p}-\left(\sum_{i=1}^{p} \lambda_{i}\right) x^{p-1}+\left(\sum_{\substack{i, j=1 \\
i \neq j}}^{p} \lambda_{i} \lambda_{j}\right) x^{p-2}-\left(\sum_{\substack{i, j, k=1 \\
i \neq j \neq k}}^{p} \lambda_{i} \lambda_{j} \lambda_{k}\right) x^{p-3}+\cdots+(-1)^{p} \lambda_{1} \lambda_{2} \cdots \lambda_{p}\right) \times \\
\left(\left(x^{n-p}-\left(\sum_{i=1}^{n-p} \lambda_{i}\right) x^{n-p-1}+\left(\sum_{\substack{i, j=1 \\
i \neq j}}^{n-p} \lambda_{i} \lambda_{j}\right) x^{n-p-2}-\left(\sum_{\substack{i, j, k=1 \\
i \neq j \neq k}}^{n-p} \lambda_{i} \lambda_{j} \lambda_{k}\right) x^{n-p-3}+\cdots+(-1)^{n-p} \lambda_{p+1} \lambda_{p+2} \cdots \lambda_{n}\right)-1\right) .
\end{gathered}
$$

Therefore, for the scalar polynomial fractional order nonlinear system in (1) with functions $f(x)$ and $g(x)$ as in (21), the fractional order sliding mode controller in (10) can be simplified as follows:

$$
u(t)=(g(x(t)))^{-1}(L(x(t))-\mu S(t)-\rho \operatorname{sgn}(s(t))) .
$$

Considering that the scalar form of $g(x(t))$, i.e., $\left(g(x(t))^{-1}\right.$, is available for $\forall x(t) \neq 0$ and as a result we can consider $h(x(t))=1$.

From item (iii) of Assumption 1, suppose that $\left\|g(x(t))^{-1}\right\| \leqslant M$. On the other hand, $\left\|g(x(t))^{-1}\right\| \geqslant$ $\frac{1}{\|g(x(t))\|}$ and according to (21) we can conclude $\|g(x(t))\|=\sqrt{\sum_{k=0}^{m}\left|b_{k}\right|^{2}}$. Therefore, $M \geqslant \frac{1}{\sqrt{\sum_{k=0}^{m}\left|b_{k}\right|^{2}}}$ and we have

$$
\sum_{k=1}^{m}\left|b_{k}\right|^{2} \geqslant \frac{1}{M^{2}}
$$

Considering $L(x(t))=x^{n}+l_{n-1} x^{n-1}+\cdots+l_{1} x+l_{0}$ and based on item (iii) of Assumption 1, $\|L(x(t))\| \leqslant N$ therefore $\sqrt{\sum_{k=0}^{n}\left|l_{k}\right|^{2}} \leqslant N$. This can also be rewritten as follows:

$$
\sum_{k=0}^{n}\left|l_{k}\right|^{2} \leqslant N^{2}
$$

In this case, the dimension of sliding surface is 1 , therefore, based on (16) the upper bound of the $u(t)$ can be obtained as follows:

$$
\|u(t)\| \leqslant M\{N+\mu+\rho\} .
$$




\section{Finite time convergence to sliding surface}

In the previous section, it has been shown that the designed sliding mode input control in (10) can asymptotically stabilize the NFO input affine system (1) and in this case the controller would remain bounded during the convergence.

In this section, it is shown that by applying the controller in (10), the system reaches the switching manifold at a specific finite time and this finite time is also obtained.

At first, consider the following two lemmas.

Lemma 1 [28,29] The relationship between the inverse hyperbolic tangent function and the logarithm function for a variable such as $\boldsymbol{x}(|\boldsymbol{x}|<1)$ is shown as follows:

$$
\tanh ^{-1}(\boldsymbol{x})=\frac{1}{2} \ln \left(\frac{1+\boldsymbol{x}}{1-\boldsymbol{x}}\right) .
$$

Lemma 2 [28,29] The relationship between the hyperbolic cosine function and the exponential function for a variable such as is shown as follows:

$$
\operatorname{sech}^{2}(x)=\left(\frac{1}{\cosh (x)}\right)^{2}=\left(\frac{2}{\mathrm{e}^{x}+\mathrm{e}^{-\boldsymbol{x}}}\right)^{2} .
$$

Theorem 2 By applying the fractional order sliding mode controller in (10) to the fractional order nonlinear system in (1), the specific finite time in which system states converge to the sliding surface is determined as follows:

$$
\begin{gathered}
t=\ln \left(\left(\frac{1}{1-\boldsymbol{S}(0)}\right)^{\frac{1}{\mu+\rho}} \times\left(\frac{1}{1+\boldsymbol{S}(0)}\right)^{\frac{-1}{\rho-\mu}} \times\right. \\
\left.\left(\frac{\rho}{\mu|\boldsymbol{S}(0)|+\rho}\right)^{\frac{2 \mu}{\rho^{2}-\mu^{2}}}\right) .
\end{gathered}
$$

Proof In order to find the reaching time to the sliding manifold, the following differential equation must be solved:

$$
\begin{gathered}
\dot{\boldsymbol{S}}(t)=\left(h(\boldsymbol{x}(t))_{0} D_{t}^{\alpha} \boldsymbol{x}(t)-h(\boldsymbol{x}(t))(f(\boldsymbol{x}(t))+L(\boldsymbol{x}(t)))\right) \times \\
\operatorname{sech}^{2}\left(\tanh ^{-1}(\boldsymbol{S}(t))\right) .
\end{gathered}
$$

Substituting (1) into (34) results in

$$
\begin{gathered}
\dot{\boldsymbol{S}}(t)=(h(\boldsymbol{x}(t))(f(\boldsymbol{x}(t))+g(\boldsymbol{x}(t)) \boldsymbol{u}(t))- \\
h(\boldsymbol{x}(t))(f(\boldsymbol{x}(t))+L(\boldsymbol{x}(t)))) \times \operatorname{sech}^{2}\left(\tanh ^{-1}(\boldsymbol{S}(t))\right) .
\end{gathered}
$$

Substituting the input control in (10) into (35) results in $\dot{\boldsymbol{S}}(t)=(-\mu \boldsymbol{S}(t)-\rho \operatorname{sgn}(\boldsymbol{S}(t))) \times \operatorname{sech}^{2}\left(\tanh ^{-1}(\boldsymbol{S}(t))\right)$.

Based on the sign of $\boldsymbol{S}(t)$ there will be two cases.
Case $1 \boldsymbol{S}(t)>0$

Assuming $\boldsymbol{S}(t)>0$, (36) can be summarized as follows:

$$
\dot{\boldsymbol{S}}(t)=(-\mu \boldsymbol{S}(t)-\rho) \times \operatorname{sech}^{2}\left(\tanh ^{-1}(\boldsymbol{S}(t))\right) .
$$

Using Lemma 2, the following equation is obtained:

$$
\begin{gathered}
\operatorname{sech}^{2}\left(\tanh ^{-1}((\boldsymbol{S}(t)))=\left(\frac{1}{\cosh \left(\tanh ^{-1}((\boldsymbol{S}(t)))\right)}\right)^{2}=\right. \\
\left(\frac{2}{\mathrm{e}^{\left(\tanh ^{-1}((\boldsymbol{S}(t)))\right.}+\mathrm{e}^{\left(\tanh ^{-1}((\boldsymbol{S}(t)))\right.}}\right)^{2} \cdot
\end{gathered}
$$

Substituting (38) into (37) results in

$$
\begin{gathered}
\dot{\boldsymbol{S}}(t)=(-\mu \boldsymbol{S}(t)-\rho) \times \\
\left(\frac{4}{\left(\mathrm{e}^{\left(\tanh ^{-1}((S(t)))\right.}+e^{\left(\tanh ^{-1}((\boldsymbol{S}(t)))\right.}\right)^{2}}\right) .
\end{gathered}
$$

Using Lemma 1, (39) could be rewritten as follows:

$$
\begin{gathered}
\dot{\boldsymbol{S}}(t)=(-\mu \boldsymbol{S}(t)-\rho) \times \\
\left(\frac{4}{\left(\mathrm{e}^{\ln \left(\frac{1+\boldsymbol{S}(t)}{1-\boldsymbol{S}(t)}\right)^{1 / 2}}+\mathrm{e}^{-\ln \left(\frac{1+\boldsymbol{S}(t)}{1-S(t)}\right)^{1 / 2}}\right)^{2}}\right) .
\end{gathered}
$$

Considering $\mathrm{e}^{\ln (\boldsymbol{x})}=\boldsymbol{x}$ that is true for any $\boldsymbol{x}$, we have

$$
\begin{gathered}
\dot{\boldsymbol{S}}(t)=(-\mu \boldsymbol{S}(t)-\rho) \times \\
\left(\frac{4}{\left(\left(\frac{1+\boldsymbol{S}(t)}{1-\boldsymbol{S}(t)}\right)^{1 / 2}+\left(\frac{1-\boldsymbol{S}(t)}{1+\boldsymbol{S}(t)}\right)^{1 / 2}\right)^{2}}\right) \\
(-\mu \boldsymbol{S}(t)-\rho) \times\left(\frac{4}{\frac{4}{1-(\boldsymbol{S}(t))^{2}}}\right)
\end{gathered}
$$

Finally we can get the following differential equation:

$$
\dot{\boldsymbol{S}}(t)=(-\mu \boldsymbol{S}(t)-\rho) \times\left(1-(\boldsymbol{S}(t))^{2}\right) .
$$

To solve the differential equation in (42), the integral must be taken from both sides.

$$
\begin{gathered}
\frac{\mathrm{d} \boldsymbol{S}(t)}{\mathrm{d} t}=(-\mu \boldsymbol{S}(t)-\rho) \times\left(1-(\boldsymbol{S}(t))^{2}\right) \\
\frac{\mathrm{d} \boldsymbol{S}(t)}{(-\mu \boldsymbol{S}(t)-\rho) \times\left(1-(\boldsymbol{S}(t))^{2}\right)}=\mathrm{d} t \\
\Rightarrow \int_{\boldsymbol{S}(0)}^{\boldsymbol{S}(t)} \frac{\mathrm{d} \boldsymbol{S}(\tau)}{(-\mu \boldsymbol{S}(\tau)-\rho) \times\left(1-(\boldsymbol{S}(\tau))^{2}\right)}=\int_{0}^{t} \mathrm{~d} \tau
\end{gathered}
$$


Using the partial decomposition technique, (45) turns out as follows:

$$
\begin{gathered}
\int_{\boldsymbol{S}(0)}^{\boldsymbol{S}(t)} \frac{\frac{-1}{\mu+\rho}}{1-\boldsymbol{S}(\tau)} \mathrm{d} \boldsymbol{S}(\tau)+\int_{\boldsymbol{S}(0)}^{\boldsymbol{S}(t)} \frac{\frac{-1}{\rho-\mu}}{1+\boldsymbol{S}(\tau)} \mathrm{d} \boldsymbol{S}(\tau)+ \\
\int_{\boldsymbol{S}(0)}^{\boldsymbol{S}(t)} \frac{\frac{-2 \mu^{2}}{\rho^{2}-\mu^{2}}}{-\mu \boldsymbol{S}(\tau)-\rho} \mathrm{d} \boldsymbol{S}(\tau)=\int_{0}^{t} \mathrm{~d} \tau .
\end{gathered}
$$

Taking the integral from (46) and doing some easy calculations result in

$$
\begin{gathered}
\ln \left(\frac{1-\boldsymbol{S}(t)}{1-\boldsymbol{S}(0)}\right)^{\frac{1}{\mu+\rho}}+\ln \left(\frac{1+\boldsymbol{S}(t)}{1+\boldsymbol{S}(0)}\right)^{\frac{-1}{\rho-\mu}}+ \\
\ln \left(\frac{-\mu \boldsymbol{S}(t)-\rho}{-\mu \boldsymbol{S}(0)-\rho}\right)^{\frac{2 \mu}{\rho^{2}-\mu^{2}}}=t .
\end{gathered}
$$

It can be rewritten as follows:

$$
\begin{gathered}
\left(\frac{1-\boldsymbol{S}(t)}{1-\boldsymbol{S}(0)}\right)^{\frac{1}{\mu+\rho}} \times\left(\frac{1+\boldsymbol{S}(t)}{1+\boldsymbol{S}(0)}\right)^{\frac{-1}{\rho-\mu}} \times \\
\left(\frac{-\mu \boldsymbol{S}(t)-\rho}{-\mu \boldsymbol{S}(0)-\rho}\right)^{\frac{2 \mu}{\rho^{2}-\mu^{2}}}=\mathrm{e}^{t} .
\end{gathered}
$$

Now, by setting $\boldsymbol{S}(t)=0$ (sliding mode) in (48), the finite time that is needed for the system to reach from the initial condition $\boldsymbol{S}(0)$ to $\boldsymbol{S}(t)=0$ is calculated as

$$
\begin{gathered}
t=\ln \left(\left(\frac{1}{1-\boldsymbol{S}(0)}\right)^{\frac{1}{\mu+\rho}} \times\left(\frac{1}{1+\boldsymbol{S}(0)}\right)^{\frac{-1}{\rho-\mu}} \times\right. \\
\left.\left(\frac{\rho}{\mu \boldsymbol{S}(0)+\rho}\right)^{\frac{2 \mu}{\rho^{2}-\mu^{2}}}\right) .
\end{gathered}
$$

Case $2 \boldsymbol{S}(t)<0$

Assuming $\boldsymbol{S}(t)<0$ we have

$$
\dot{\boldsymbol{S}}(t)=(-\mu \boldsymbol{S}(t)+\rho) \times \operatorname{sech}^{2}\left(\tanh ^{-1}(\boldsymbol{S}(t))\right) .
$$

By performing the similar process, the zeroing time of sliding surface for $\boldsymbol{S}(t)<0$ is obtained as follows:

$$
\begin{gathered}
t=\ln \left(\left(\frac{1}{1-\boldsymbol{S}(0)}\right)^{\frac{1}{\mu+\rho}} \times\left(\frac{1}{1+\boldsymbol{S}(0)}\right)^{\frac{-1}{\rho-\mu}} \times\right. \\
\left.\left(\frac{\rho}{-\mu \boldsymbol{S}(0)+\rho}\right)^{\frac{2 \mu}{\rho^{2}-\mu^{2}}}\right) .
\end{gathered}
$$

Thus, according to (49) and (51), the finite time in which system states converge to the sliding manifold can be expressed as follows:

$$
t=\ln \left(\left(\frac{1}{1-\boldsymbol{S}(0)}\right)^{\frac{1}{\mu+\rho}} \times\left(\frac{1}{1+\boldsymbol{S}(0)}\right)^{\frac{-1}{\rho-\mu}} \times\right.
$$

$$
\left.\left(\frac{\rho}{\mu|\boldsymbol{S}(0)|+\rho}\right)^{\frac{2 \mu}{\rho^{2}-\mu^{2}}}\right)
$$

\section{Comparison with integer order counterpart}

In this section, a comparison between fractional order sliding mode control in (10) with its integer order counterpart is obtained, before that we first express some lemmas.

Lemma 3 [30] Consider a nonlinear fractional order equation as follows:

$$
D_{t}^{\alpha} \boldsymbol{y}(t)=\boldsymbol{F}(t) .
$$

Then, the solution of (53) is

$$
\boldsymbol{y}(t)=\sum_{n=0}^{\infty} \frac{\boldsymbol{F}^{n}(0)}{n !} \frac{\Gamma(n+1)}{\Gamma(1+n+\alpha)} t^{n+\alpha}=D_{t}^{-\alpha} \boldsymbol{F}(t) .
$$

And also the solution of the one-term equation in (53) can be generalized when the right-hand side of the equation in (53) has other forms [30]. Suppose that $F$ is expressed as follows:

$$
\boldsymbol{F}(\boldsymbol{x}(t))=f(\boldsymbol{x}(t))+L(\boldsymbol{x}(t)) .
$$

According to (9), when the system is in the siding mode, it can be shown as follows:

$$
D_{t}^{\alpha} \boldsymbol{x}(t)=f(\boldsymbol{x}(t))+L(\boldsymbol{x}(t)) .
$$

Lemma 4 [31] Let $\boldsymbol{x}(t)=0$ be an equilibrium point of the system in (56), $f+L$ is Lipschitz on $\boldsymbol{x}(t)=0$ with Lipschitz constant $l$ and is also piecewise continuous with respect to $t$, then the solution of (56) satisfies

$$
\|\boldsymbol{x}(t)\| \leqslant\left\|\boldsymbol{x}_{0}(t)\right\| E_{\alpha}\left(l\left(t-t_{0}\right)^{\alpha}\right)
$$

where $\boldsymbol{x}_{0}(t)$ is the initial condition and $E_{\alpha}(\cdot)$ is MittagLeffler function.

Remark 2 [31] In Lemma 4, if $\alpha=1$, it follows [32] that

$$
\|\boldsymbol{x}(t)\| \leqslant\left\|\boldsymbol{x}_{0}(t)\right\| \mathrm{e}^{l\left(t-t_{0}\right)} .
$$

In comparison to the integer order counterpart system that is $\dot{\boldsymbol{x}}(t)=f(\boldsymbol{x}(t))+L(\boldsymbol{x}(t))$, the state $\boldsymbol{x}(t)$ of the fractional order system decays to 0 like $t^{-\alpha}$ whereas the states in the integer order case decay towards 0 like $\mathrm{e}^{A t}, A=$ $\frac{\partial}{\partial \boldsymbol{x}(t)}(f(\boldsymbol{x}(t))+L(\boldsymbol{x}(t)))$.

As it is shown in [33], this means that from the initial state $\boldsymbol{x}_{0}(t)$, the chattering layer of the fractional order nonlinear system around switching manifold $\boldsymbol{S}(t)=0$ is smaller than that of the integer order counterpart. Fig. 1 
shows this difference for a system with two states. When the system reaches the siding surface from initial states $\left(x_{10}, x_{20}\right)$, for the fractional order system, states decay toward $(0,0)$ like $t^{-\alpha}(1 \rightarrow 2 \rightarrow 3 \ldots)$ whereas the states in integer order case decays to origin like $\mathrm{e}^{A t}\left(1^{\prime} \rightarrow 2^{\prime} \rightarrow\right.$ $\left.3^{\prime} \ldots\right)$.

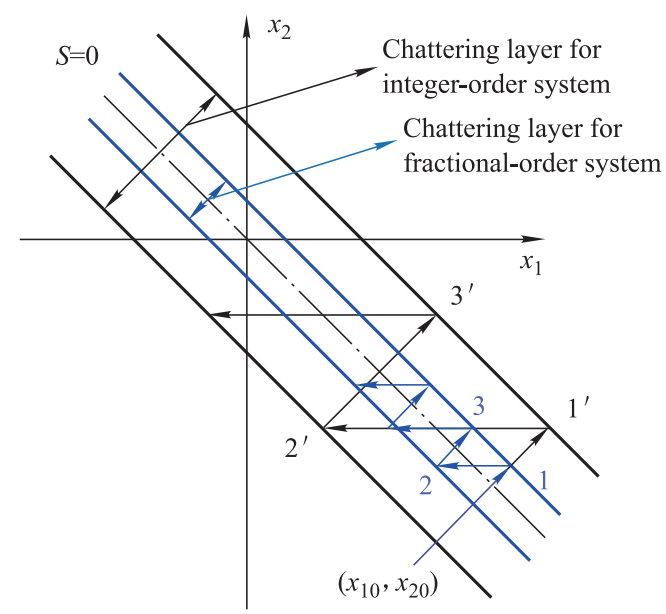

Fig. 1 Decaying of states on sliding mode surface in fractional-order compared with the integer order systems

This clearly shows that chattering in fractional order sliding mode control is less than of it for integer order counterpart and this is one of the advantages of fractional order sliding mode control compared with classical integer order systems.

\section{Simulation results}

In this section some simulation results are presented for nonlinear fractional order input affine systems with the designated bounded fractional order sliding mode controller to show the effectiveness of the proposed methodology.

\section{Example 1}

Based on the financial chaotic systems which are provided in [34,35], functions $f(\boldsymbol{x}(t)), g(\boldsymbol{x}(t))$ for the system (1) are defined as follows:

$$
\left\{\begin{aligned}
& f(\boldsymbol{x}(t))= {\left[\begin{array}{c}
x_{1}(t) x_{2}(t) \\
-x_{1}^{2}(t) \\
x_{2}(t) x_{3}(t)
\end{array}\right] } \\
& g(\boldsymbol{x}(t))=\left[\begin{array}{c}
1 \\
2+\cos \left(x_{1}(t) x_{2}(t)\right) \\
1
\end{array}\right] .
\end{aligned}\right.
$$

As previously mentioned, $L(\boldsymbol{x}(t))$ and $H(\boldsymbol{x}(t))$ are two arbitrary functions that should satisfy conditions of Assump- tion 1 . Thus they can be chosen as follows:

$$
\left\{\begin{array}{l}
L(\boldsymbol{x}(t))=\left[\begin{array}{l}
-x_{3}^{2}(t) \\
-x_{2}(t) \\
-x_{1}(t)
\end{array}\right] \\
H(\boldsymbol{x}(t))=\left[\begin{array}{c}
2 \\
\tanh \left(x_{1}(t)\right) \\
10
\end{array}\right] .
\end{array}\right.
$$

The initial condition of the system, fractional order and designed gains for the controller are selected as $x_{0}=$ $\left[\begin{array}{lll}2 & -1 & 1\end{array}\right]^{\mathrm{T}}, \alpha=0.98, \rho=2, \mu=5$, respectively.

Based on the designed sliding mode controller in (10), the simulation results are shown in Fig. 2 and Fig. 3. It is clearly seen in Fig. 2 that the designed sliding mode controller is capable for stabilizing the system in (59) as well. The designed sliding mode controller is shown in Fig. 3. In Fig. 3, it is seen that the designed controller is bounded between -0.6242 and 0.6242 . The constrained diagram of the sliding surface and its convergence to zero are shown in Fig. 4. Fig. 4 shows that the sliding surface converges to zero in a finite time that is $2.04 \mathrm{~s}$.

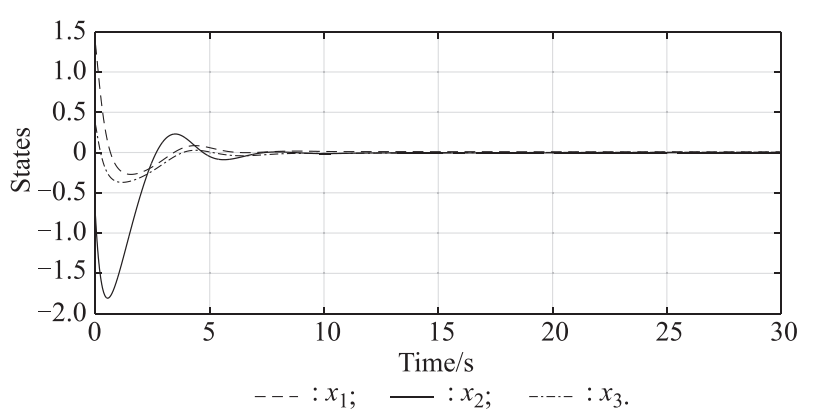

Fig. 2 State convergence to the origin

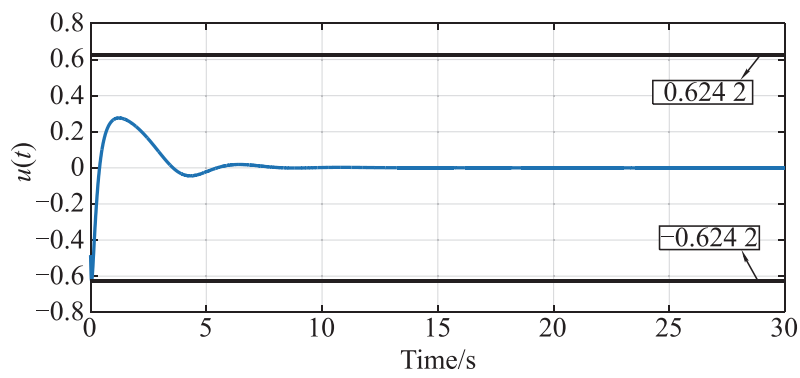

Fig. 3 Designed constrained control input for the system by sliding mode method

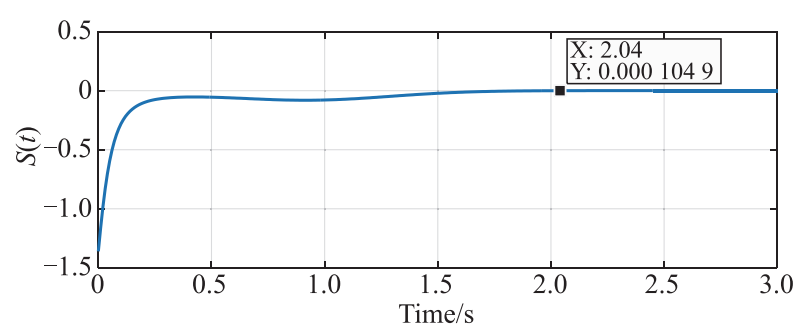

Fig. 4 Designed constrained sliding surface and its convergence to zero 
The designed constrained fractional order controller has been compared with its counterpart integer order one. To do this, both of these controllers have been applied to an integer order system. The result has been shown in Fig. 5. From Fig. 5, it is seen that the fractional order controller has better performance than the integer order counterpart. It is clearly shown that the fractional order controller results in fewer chattering in convergence of states.

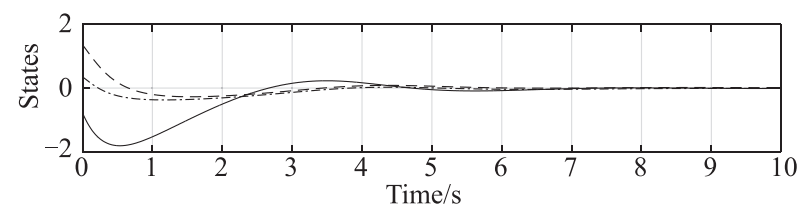

(a) Integer order system with fractional order controller

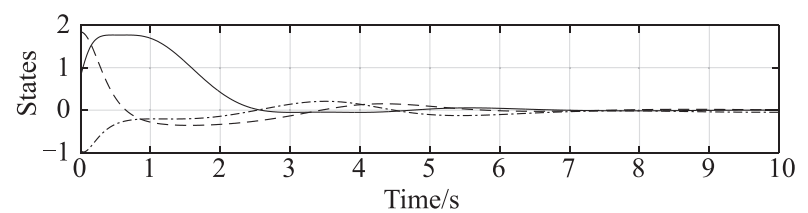

(b) Integer order system with integer order controller

$$
---: x_{1} ; \quad-: x_{2} ; \quad-\ldots-x_{3} .
$$

Fig. 5 Comparison between integer order and fractional order controller which are applied to the integer order system

\section{Example 2}

In this example functions $f(x)$ and $g(x)$ are considered as scalar polynomials and also we have $\alpha=0.95, \mu=3$, $\rho=2$. Assume that $f(x)$ and $g(x)$ are as follows:

$$
\left\{\begin{array}{l}
f(x(t))=3 x^{3}+x^{2}+x+1 \\
g(x(t))=2 x^{2}+x+1
\end{array}\right.
$$

where $f(x(t))$ has three roots which are $\lambda_{1,2}=0.1508 \pm$ j0.708 $6, \lambda_{3}=-0.635$. It is known that $\arg (x, y)=$ $\arctan \left(\frac{y}{x}\right)+\frac{\pi}{2} \operatorname{sign}(y)(1-\operatorname{sign}(x))$, hence the absolute argument of the roots can be obtained as follows:

$$
\left\{\begin{array}{l}
\left|\arg \left(\lambda_{1,2}\right)\right|=1.3611<\alpha \frac{\pi}{2}, \\
\left|\arg \left(\lambda_{3}\right)\right|=\pi>\alpha \frac{\pi}{2}
\end{array} .\right.
$$

It is obvious that the nonlinear function $f(x(t))$ has one stable and two unstable roots. Based on (26), function $L(x(t))$ can be designed as follows:

$$
L(x(t))=-x^{3}-0.3334 x^{2}+0.6667 x+0.3017 .
$$

Now, by calculating $M$ and $N$ from (28) and (29) we have

$$
M \geqslant \frac{1}{\sqrt{\sum_{k=0}^{m}\left|b_{k}\right|^{2}}}=\frac{1}{\sqrt{(2)^{2}+(1)^{2}+(1)^{2}}}=\frac{1}{\sqrt{6}}
$$

$$
\begin{gathered}
N^{2} \geqslant \sum_{k=0}^{n}\left|l_{k}\right|^{2}=1^{2}+0.3334^{2}+0.6667^{2}+ \\
0.3017^{2}=1.645 \Rightarrow N \geqslant 1.2832 .
\end{gathered}
$$

Thus the upper bound of the input control from (30) could be $|u(t)| \leqslant \frac{1}{\sqrt{6}}(1.2832+3+2)=2.5651$.

Fig. 6 shows the system state. It is seen that the state smoothly converges to the origin.

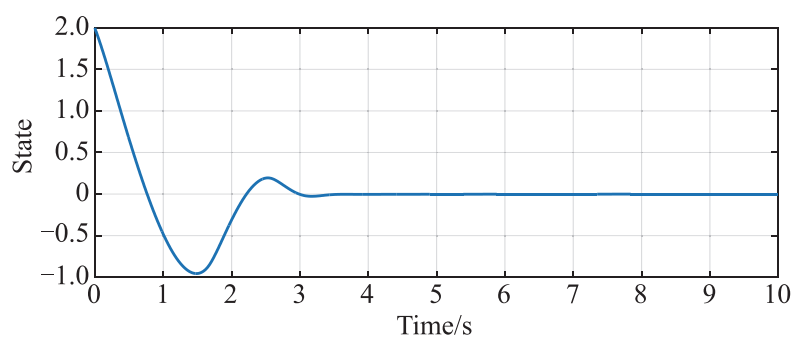

Fig. 6 Scalar fractional order controlled system

The bounded input control is shown in Fig. 7. It can be seen that the input control remains in the designed bound that is 2.5651 .

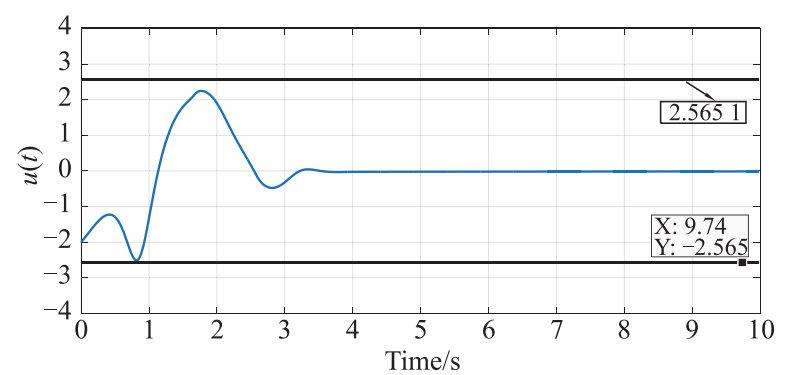

Fig. 7 Input control and its boundaries

\section{Example 3}

One of the fractional order chaotic systems which is used in cryptography is the fractional order Lorenz system [36]. An analog circuit to implement this system by using resistors, capacitors, analog multipliers AD633, and analog operational amplifiers LF347N, is shown in Fig. 8. That is, the dynamics of the system are confirmed by physical implementation [37].

Equations of the fractional order Lorenz system are listed as follows:

$$
\left\{\begin{array}{l}
D^{\alpha} x=\sigma(y-x) \\
D^{\alpha} y=\gamma x-y-x z \\
D^{\alpha} z=x y-\beta z
\end{array}\right.
$$

where $\sigma=10, \rho=28, \beta=\frac{8}{3}$ and $\alpha=0.97, \mu=$ $2, \rho=3$. Initial conditions for this system are considered as $\left[x_{0}, y_{0}, z_{0}\right]^{\mathrm{T}}=[0.5,-1,0.8]^{\mathrm{T}}$. The chaotic behavior of this system is shown in Fig. 9. 

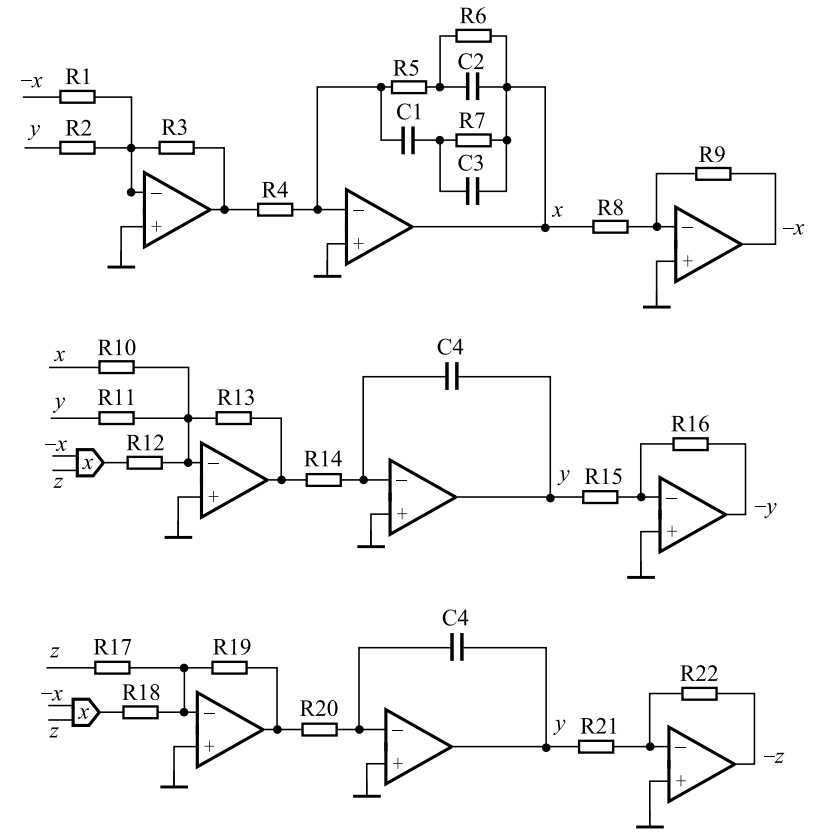

Fig. 8 Analog circuit of the fractional-order Lorenz system

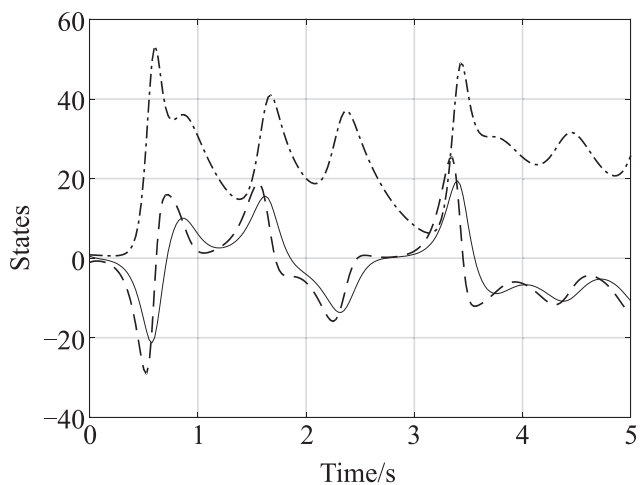

(a) Trajectories of Lorenz system

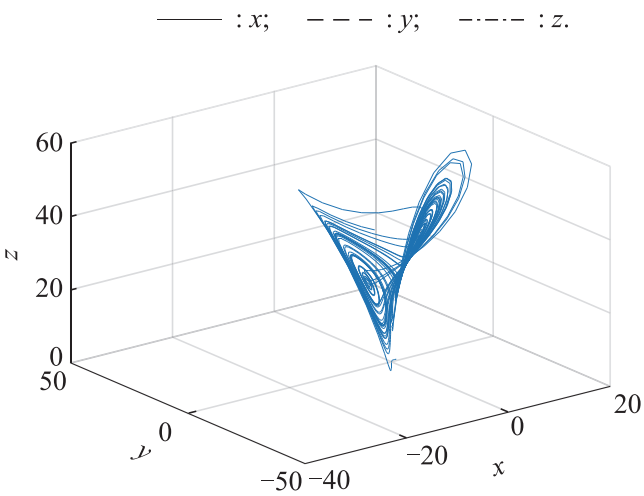

(b) $3 \mathrm{D}$ chaotic Lorenz

Fig. 9 Chaotic fractional Lorenz system

To stabilize the system, a limited input control is applied to the second state. Thus, the system equation is listed as follows:

$$
\left\{\begin{array}{l}
D^{\alpha} x=\sigma(y-x) \\
D^{\alpha} y=\rho x-y-x z+u \\
D^{\alpha} z=x y-\beta z
\end{array} .\right.
$$

Therefore, $\boldsymbol{f}(x), \boldsymbol{g}(x)$ and designed $\boldsymbol{L}(x), \boldsymbol{H}(x)$ are listed as follows:

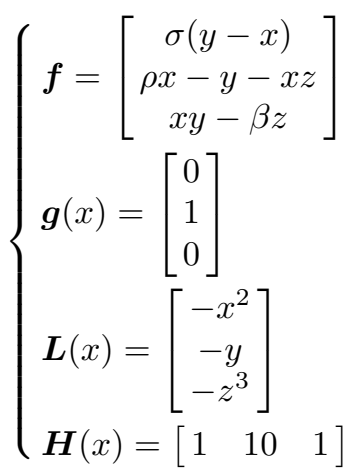

Based on designed sliding mode controller in (10), the simulation results are shown in Fig. 10 and Fig. 11. From Fig. 10, it is seen that the designed sliding mode controller is capable for stabilizing the system in (66) and system states will converge to the origin after about $1.7 \mathrm{~s}$. The designed sliding mode controller is shown in Fig. 11. It is seen that the designed controller is bounded between -1 and 1 . The constrained diagram of sliding surface and its convergence to zero are shown in Fig. 12. Fig. 12 shows that the sliding surface converges to zero in a finite time that is $1.7 \mathrm{~s}$.

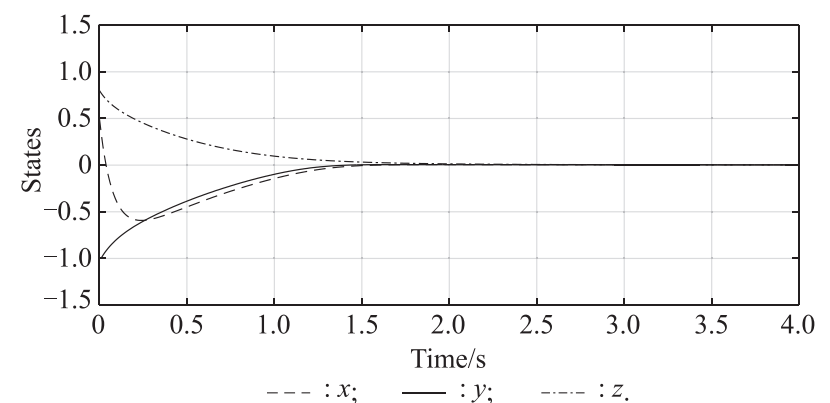

Fig. 10 Lorenz state convergence to the origin

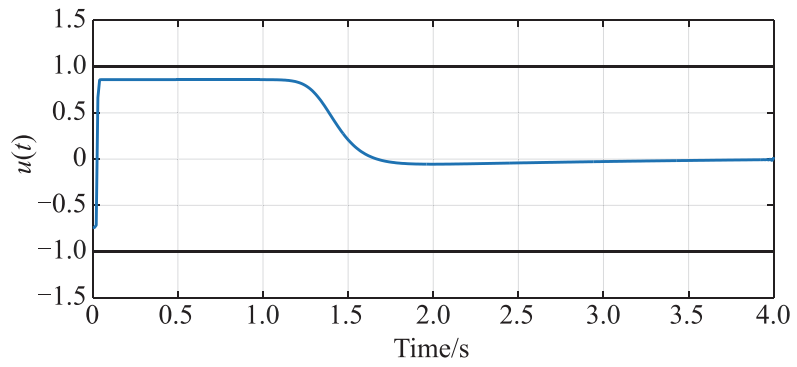

Fig. 11 Designed constrained control input for the Lorenz system

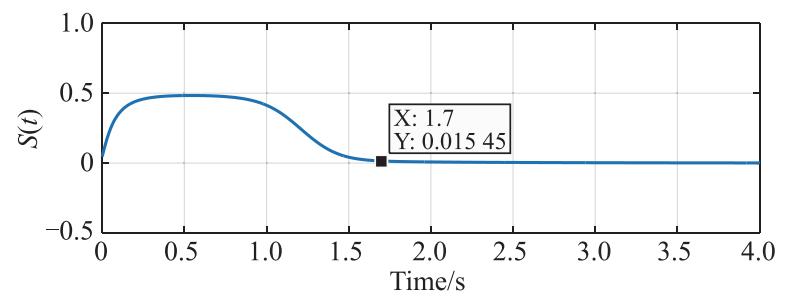

Fig. 12 Designed constrained sliding surface for the Lorenz system 


\section{Conclusions}

In this paper a new bounded and chattering free fractional order sliding mode controller is designed for nonlinear fractional order input affine systems. In order to limit the input controller and reduce the chattering, a hyperbolic tangent function is used in designing the sliding surface. Using this sliding mode controller, asymptotic stability of the closed loop system is obtained and the input controller is remained bounded. In addition, the finite time convergence of the sliding surface to zero is calculated, stability of polynomial nonlinear fractional order systems as a special case is presented and finally a comparison with the integer order counterpart is also presented. The simulation results confirm the obtained mathematical relationships.

\section{References}

[1] LI Y M, TONG S C, LI T S. Composite adaptive fuzzy output feedback control design for uncertain nonlinear strict-feedback systems with input saturation. IEEE Trans. on Cybernetics, 2014, 45(10): 2299-2308.

[2] YANG Y, YUE D, XUE Y S. Decentralized adaptive neural output feedback control of a class of large-scale time-delay systems with input saturation. Journal of the Franklin Institute, 2015, 352(5): 2129-2151.

[3] GOUTA H, SAID S H, SAHLI F M. Model-based predictive and backstepping controllers for a state coupled four-tank system with bounded control inputs: a comparative study. Journal of the Franklin Institute, 2015, 352(11): 4864-4889.

[4] AZINHEIRA J R, MOUTINHO A. Hover control of an UAV with backstepping design including input saturations. IEEE Trans. on Control Systems Technology, 2008, 16(3): $517-$ 526.

[5] WANG H Q, CHEN B, LIU X P, et al. Adaptive neural tracking control for stochastic nonlinear strict-feedback systems with unknown input saturation. Information Sciences, 2014, 269(10): $300-315$.

[6] LI Y M, TONG S C, LI T S. Adaptive fuzzy output-feedback control for output constrained nonlinear systems in the presence of input saturation. Fuzzy Sets \& Systems, 2014, 248: $138-155$.

[7] LI Y M, TONG S C, LI T S. Hybrid fuzzy adaptive output feedback control design for uncertain MIMO nonlinear systems with time-varying delays and input saturation. IEEE Trans. on Fuzzy Systems, 2016, 24(4): 841-853.

[8] LI Y M, TONG S C, LI T S. Observer-based adaptive fuzzy tracking control of MIMO stochastic nonlinear systems with unknown control direction and unknown dead-zones. IEEE Trans. on Fuzzy Systems, 2015, 23(4): $1228-1241$.

[9] TEEL A R, PRALY L. On assigning the derivative of a disturbance attenuation control lyapunov function. Mathematics of Control, Signals and Systems, 2000, 13(2): 95-124.

[10] LOGEMANN H, RYAN E. Time-varying and adaptive discrete-time low-gain control of infinite-dimensional linear systems with input nonlinearities. Mathematics of Control, Signals and Systems, 2000, 13(4): 293-317.

[11] NOROOZI N, KHAYATIAN A, GEISELHART R. A characterization of integral input-to-state stability for hybrid systems. Mathematics of Control, Signals and Systems, 2017, 29(3): $1-32$.

[12] LIM Y H, OH K K, AHN H S. Stability and stabilization of fractional-order linear systems subject to input saturation. IEEE Trans. on Automatic Control, 2013, 58(4): 1062 - 1067.

[13] SHAHRI E S A, ALFI A, MACHADO J A T. An extension of estimation of domain of attraction for fractional order linear system subject to saturation control. Applied Mathematics Letters, 2015, 47: 26-34.

[14] SHAHRI E S A, BALOCHIAN S. Analysis of fractionalorder linear systems with saturation using Lyapunov's second method and convex optimization. International Journal of Automation and Computing, 2015, 12(4): 440-447.

[15] LUO J H. State-feedback control for fractional-order nonlinear systems subject to input saturation. Mathematical Problems in Engineering, 2014, DOI:10.1155/2014/891639.

[16] UTKIN V, GULDNER J, SHI J. Sliding mode control in electro-mechanical systems. Boca Raton: CRC Press, 2009.

[17] EDWARDS C, SPURGEON S K. Sliding mode control: theory and applications. Boca Raton: CRC Press, 1998.

[18] UTKIN V I. Sliding modes in control and optimization. Berlin Heidelberg: Springer-Verlag, 1992.

[19] FRIDMAN L, LEVANT A. Sliding mode control enginerring. Boca Raton: CRC Press, 2002.

[20] SI-AMMOUR A, DJENNOUNE S, BETTAYEB M. A sliding mode control for linear fractional systems with input and state delays. Communications in Nonlinear Science and Numerical Simulation, 2009, 14(5): 2310-2318.

[21] DADRAS S, MOMENI H R. Fractional terminal sliding mode control design for a class of dynamical systems with uncertainty. Communications in Nonlinear Science and Numerical Simulation, 2012, 17: $367-377$.

[22] MUJUMDAR A, KURODE S, TAMHANE B. Fractionalorder sliding mode control for single link flexible manipulator. Proc. of the IEEE International Conference on Control Applications, 2013: $288-293$.

[23] ZHANG D, CAO L, TANG S. Fractional-order sliding mode control for a class of uncertain nonlinear systems based on LQR. International Journal of Advanced Robotic Systems, 2017, 14(2): 1-15.

[24] MÖ E F E. New trends in nanotechnology and fractional calculus applications. Dordrecht: Springer, 2010.

[25] RAMÍREZ H S, BATTLE V F. Modern sliding mode control theory. Berlin: Springer, 2008.

[26] PISANO A, RAPAI'C M, USAI E. Sliding modes after the first decade of the 21st century: state of the art. Berlin: Springer Science and Business Media, 2011.

[27] LI C P, ZHANG F R. A survey on the stability of fractional differential equations. European Physical Journal Special Topics, 2011, 193: $27-47$.

[28] WOODHOUSE N M J. Special relativity. London: SpringerVerlag, 2003.

[29] ULRICH L, ROHDE G C J, PODDAR A K, et al. Introduction to differential calculus: systematic studies with engineering applications for beginners. New York: Wiley, 2012.

[30] PODLUBNY I. Fractional differential equations: an introduction to fractional derivatives, fractional differential equations, to methods of their solution and some of their applications. Salt Lake: Academic Press, 1998.

[31] LI Y, CHEN Y Q, PODLUBNY I. Stability of fractional-order nonlinear dynamic systems: Lyapunov direct method and generalized mittag-leffler stability. Computers and Mathematics with Applications, 2010, 59(5): $1810-1821$.

[32] SASTRY S, BODSON M. Adapative control-stability convergence and robustness. New Jersey: Prentice Hall, 1989.

[33] ZHANG B T, PI Y G, LUO Y. Fractional order sliding-mode control based on parameters auto-tuning for velocity control of permanent magnet synchronous motor. ISA Transactions, 
2012, 51(5): $649-656$.

[34] CHEN W C. Nonlinear dynamics and chaos in a fractionalorder financial system. Chaos, Solitons and Fractals, 2008, 36(5): $1305-1314$.

[35] ZHEN W, XIA H, GUODONG S. Analysis of nonlinear dynamics and chaos in a fractional order financial system with time delay. Computers and Mathematics with Applications, 2011, 62(3): $1531-1539$.

[36] JIANG Z P. A note on chaotic secure communication systems. IEEE Trans. on Circuits Systerm I-Fundamental Theory and Applications, 2002, 49(1): 92-96.

[37] JIA H Y, TAO Q, CHEN Z Q. Analysis and circuit design of a fractional-order Lorenz system with different fractional orders. Systems Science \& Control Engineering, 2014, 2(1): $745-$ 750.

\section{Biographies}

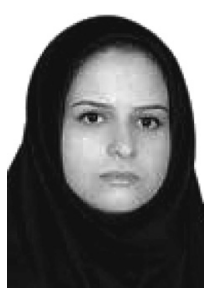

TAHMINEH Vedadi Moghaddam was born in 1985. She received her B.S. degree in 2007 from Imam Khomeini International University, Qazvin, Iran. She received her M.S. degree in 2011, in the field fractional order controller, from Electrical Engineering Department of Imam Khomeini International University, Qazvin, Iran. Currently, she is working towards her Ph.D. degree at Amirkabir University of Technology, Tehran, Iran. Her current research interests include robust control, adaptive control and nonlinear fractional order systems.

Email: t_vedadi@aut.ac.ir

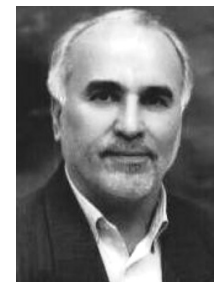

SEYYED KAMALEDDIN Yadavar Nikravesh was born in 1944. He received his B.S. degree in 1967 from Electrical Engineering Department of Tehran Polytechnic University, Tehran, Iran. He received his M.S. degree in 1971, in the field of major option in control, from Electrical Engineering Department of University of Missouri, Rolla, U.S.A. He received his Ph.D. degree in 1973, in the field of major option in control, from Electrical Engineering, University of Missouri, Columbia, U.S.A. He taught over the past 40 years as a professor at the Amirkabir University of Technology, Tehran, Iran. His current research interests include optimal control theory, electrical circuit analysis, industrial control systems and nonlinear control theory.

Email: nikravsh@aut.ac.ir

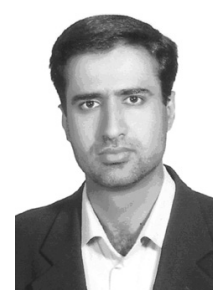

MOHAMMAD Azam Khosravi was born in 1976. He received his M.Sc.and his Ph.D. degrees from K.N. Toosi University of Technology, Tehran, Iran, in 2000 and 2013, respectively, all in electrical engineering. He is currently an assistant professor with Amirkabir University of Technology. His research interests include nonlinear control theory, parallel robotics, cable driven robots and robust control. Email: m.a.khosravi@aut.ac.ir 